1. Studer SM, Levy RD, McNeil K, Orens JB. Lung transplant outcomes: a review of survival, graft function, physiology, health-related quality of life and cost-effectiveness. Eur Respir J. 2004;24(4):674-685.

2. Shilling RA, Wilkes DS. Immunobiology of chronic lung allograft dysfunction: new insights from the bench and beyond. Am J Transplant. 2009; 9(8):1714-1718.

3. Dhillon GS, et al. Lung transplant airway hypoxia: a diathesis to fibrosis? Am J Respir Crit Care Med. 2010;182(2):230-236.

4. Luckraz H, et al. Microvascular changes in small airways predispose to obliterative bronchiolitis after lung transplantation. J Heart Lung Transplant. 2004;23(5):527-531.

5. Luckraz H, Goddard M, McNeil K, Atkinson C, Sharples LD, Wallwork J. Is obliterative bronchiolitis in lung transplantation associated with microvascular damage to small airways? Ann Thorac Surg. 2006;82(4):1212-1218.

6. Zhou G, et al. Hypoxia-induced alveolar epithelialmesenchymal transition requires mitochondrial ROS and hypoxia-inducible factor 1. Am J Physiol Lung Cell Mol Physiol. 2009;297(6):L1120-L1130.

7. Willis BC, Borok Z. Epithelial-mesenchymal tran- sition: potential role in obliterative bronchiolitis? Thorax. 2009;64(9):742-743.

8. Borthwick LA, et al. Epithelial to mesenchymal transition (EMT) and airway remodelling after human lung transplantation. Thorax. 2009;64(9):770-777.

9. Ward C, et al. Phenotype of airway epithelial cells suggests epithelial to mesenchymal cell transition in clinically stable lung transplant recipients. Tho$\operatorname{rax} .2005 ; 60(10): 865-871$.

10. Gelman AE, et al. Cutting edge: Acute lung allograft rejection is independent of secondary lymphoid organs. J Immunol. 2009;182(7):3969-3973.

11. Constant SL, et al. Resident lung antigen-presenting cells have the capacity to promote Th2 T cell differentiation in situ. JClin Invest. 2002;110(10):1441-1448.

12. Kamler M, et al. Bronchial artery revascularization restores peribronchial tissue oxygenation after lung transplantation. J Heart Lung Transplant. 2004;23(6):763-766.

13. Wang $Q$, et al. Reoxygenation of hypoxia-differentiated dentritic cells induces Th1 and Th17 cell differentiation. Mol Immunol. 2010;47(4):922-931.

14. Jantsch J, et al. Hypoxia and hypoxia-inducible factor- 1 alpha modulate lipopolysaccharide-induced dendritic cell activation and function. J Immunol.
2008;180(7):4697-4705

15. Burlingham WJ, et al. IL-17-dependent cellular immunity to collagen type $\mathrm{V}$ predisposes to obliterative bronchiolitis in human lung transplants. J Clin Invest. 2007;117(11):3498-3506.

16. Jiang X, et al. Adenovirus-mediated HIF- $1 \alpha$ gene transfer promotes repair of mouse airway allograft microvasculature and attenuates chronic rejection. J Clin Invest. 2011;121(6):2336-2349.

17. Babu AN, et al. Microvascular destruction identifies murine allografts that cannot be rescued from airway fibrosis. J Clin Invest. 2007;117(12):3774-3785.

18. Belperio JA, et al. Critical role for CXCR3 chemokine biology in the pathogenesis of bronchiolitis obliterans syndrome. JImmunol. 2002;169(2):1037-1049.

19. Rey S, Semenza GL. Hypoxia-inducible factor-1dependent mechanisms of vascularization and vascular remodelling. Cardiovasc Res. 2010;86(2):236-242.

20. Bosch-Marce M, et al. Effects of aging and hypoxiainducible factor- 1 activity on angiogenic cell mobilization and recovery of perfusion after limb ischemia. Circ Res. 2007;101(12):1310-1318.

21. Fan L, et al. Neutralizing IL-17 prevents obliterative bronchiolitis in murine orthotopic lung transplantation. Am J Transplant. 2011;11(5):911-922.

\title{
The yin, the yang, and the Angiopoietin-1
}

\section{Pipsa Saharinen and Kari Alitalo}

Molecular/Cancer Biology Program and Finnish Institute for Molecular Medicine, University of Helsinki, Helsinki, Finland.

\begin{abstract}
Twenty years after the discovery of the vascular endothelial Tie receptor tyrosine kinases and 15 years after the discovery of the Tie2 ligand, angiopoietin-1 (Angpt1, also known as Ang1), a study published in the current issue of the JCI reveals an unexpected loss-of-function phenotype of mice conditionally deleted of the Angpt1 gene. The results suggest that Angpt1 is needed as a vascular stabilizing factor that organizes and limits the angiogenesis response and protects from pathological consequences, such as tissue fibrosis.
\end{abstract}

\section{Angiopoietin-1 and angiopoietin-2 in sprouting angiogenesis}

In the process of sprouting angiogenesis that typically occurs when VEGF stimulates capillaries, the leading edges of growing capillary sprouts display migrating and slowly dividing tip cells, which extend filopodia in response to VEGF gradients (1). Tip cells are followed by less mobile proliferating stalk cells, which express the Tie2 receptor and recruit pericytes. Angiopoietin-1 (Angpt1) is expressed by perivascular cells, such as pericytes, and the related Tie2 ligand Angpt2 is expressed by the endothelial tip cells. Although Angpt 1 and Angpt 2 both bind to Tie2 with similar affinities, they seem to act as antagonists of each other in several vascular processes

Conflict of interest: The authors declare research grant support from Medimmune, LLC.

Citation for this article: J Clin Invest. 2011; 121(6):2157-2159. doi:10.1172/JCI58196.
$(2,3)$. In the stalk cells that become associated with pericytes, Angpt 1 may limit angiogenesis by inducing homomeric Tie2 complexes across the cell-cell junctions, and mediating cell-cell adhesion, antipermeability, and cell survival $(4,5)$. In contrast, Angpt2 may regulate cell-matrix interactions in the growing vessels to facilitate sprouting (Figure 1).

\section{Myocardial Angpt1 is essential for cardiovascular development}

The study by Jeansson et al. shows that despite the constitutive expression of Angpt 1 in many cell types, including perivascular mural cells, and constitutive Tie2 activation in vascular endothelial cells in vivo $(2,6)$, Angpt 1 is not necessary for normal steady-state physiological processes in the adult, being dispensable in the blood vasculature from E13.5 onward (7). However, Angpt 1 is essential for the development of embryonic vasculature during a short period between E10.5 and E13.5 $(7,8)$. Tie 2 and the related Tie1 (9) are also critical during that period. Analysis of Tie2-deleted and mosaic mutant embryos at E10.5 indicated that Tie 2 is required in the developing endocardium for myocardial attachment and trabeculation, whereas Tie 2 and Tie 1 are dispensable for the initial assembly of the rest of the vasculature (10-12). Thus, it was of no surprise that the embryos died when, in the study by Jeansson et al., Angpt1 was deleted from the developing embryos before E12.5 (7). Interestingly, however, these new data indicate that cardiomyocyte-specific deletion of Angpt1 reproduces much of the phenotype of the full Angpt1 knockout, suggesting that hemodynamic problems propagate the vascular defects to other tissues $(7,8)$. However, studies in which Angpt 1 would be deleted in other developing vascular beads, while leaving the cardiac Angpt1 levels intact, are needed to confirm these results. The previously published mosaic analysis indicated that both Tie 1 and Tie 2 are required in the microvasculature during late organogenesis and in essentially all blood vessels of the adult (12, 13). The report by Jeansson et al. thus raises the intriguing possibility that the requirement for Tie 1 and Tie2 in the microvasculature is independent of Angpt1 function. 
Stable blood vessel

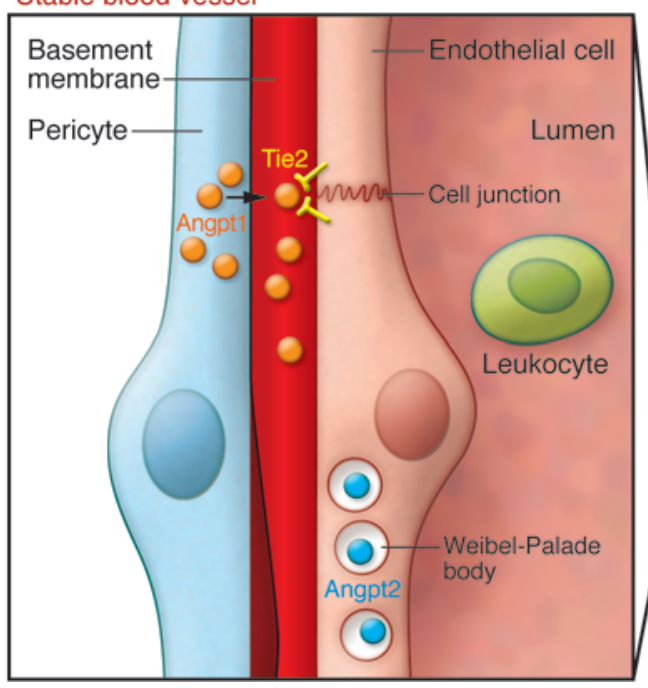

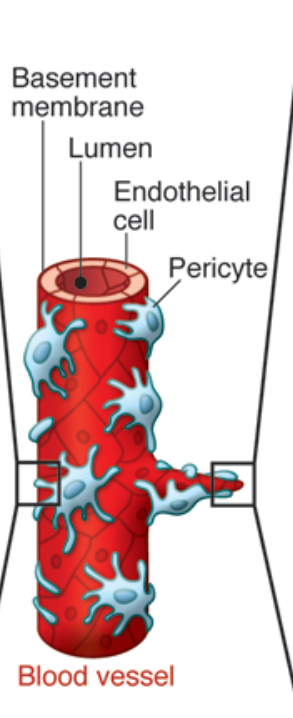

Angiogenic blood vessel

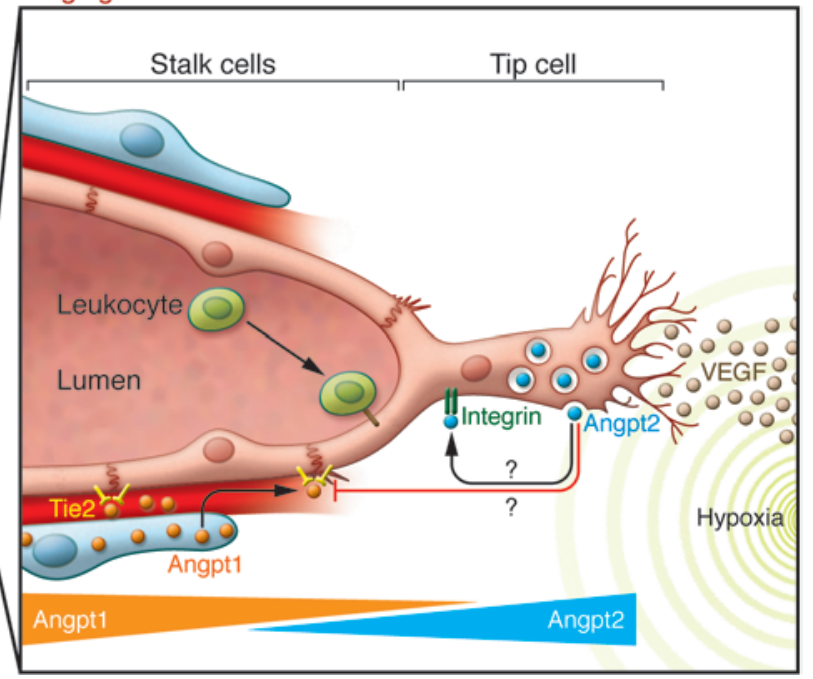

\section{Figure 1}

The Angpt-Tie system in stable vessels and sprouting angiogenesis. In stable vessels, Angpt1 is produced by pericytes, Tie 2 is activated in endothelial cells, and Angpt2 is stored in Weibel-Palade bodies. However, the data by Jeansson et al. in this issue of the $\mathrm{JCl}$ indicates that Angpt 1 function is not necessary for normal vascular physiology (7), although Tie2 has been found to be constitutively phosphorylated in quiescent endothelium (6). In vessels undergoing angiogenesis in response to VEGF secreted by nearby hypoxic cells, Angpt2 is expressed predominantly in the tip cells of angiogenic sprouts, where it may regulate cell-matrix interactions by binding to integrins and connective tissue matrix. The Tie2 receptor is expressed in the stalk cells, which become coated with pericytes and the basement membrane matrix that accumulates in between the cells in the stabilization phase of angiogenesis. Angpt1, from the perivascular cells, interacts with the Tie2 receptor. In this context, Angpt 1 is necessary for the stabilization of the newly formed vessels, for attenuation of angiogenesis, and for limiting the production of excess of connective tissue. Whether Angpt2 counteracts the Angpt1-induced Tie2 activation in the stalk cells located behind the angiogenic tip cell area is as yet not clear. Angpt2 may also facilitate leukocyte adhesion to the endothelium of newly forming sprouts.

Pericytes were normally invested around the endothelium in resting and angiogenic capillaries of the Angpt1-deleted mice (7), in line with studies of Tie2 $2^{-/}$Tie1 $1^{-/}$chimeric embryos, which do not show differences in pericyte recruitment (12). Angpt2 made by endothelial cells is stored in Weibel-Palade bodies, from which it is rapidly released in various pathological processes (14), facilitating endothelial cell responsiveness to inflammatory and angiogenic cytokines. Autocrine Angpt2 secretion is also associated with pericyte detachment (2). The report by Jeansson et al. suggests that Angpt2induced pericyte loss might not be due to its inhibition of Angpt1 function (7). Exogenous Angpt1 is known to inhibit vessel permeability, especially when induced by a variety of cytokines (15). However, Jeansson et al. reveal in their discussion that, in their model, Angpt1 could be deleted without effects on the permeability of mature, quiescent vessels (7).

\section{Angpt1 deletion leads to excessive angiogenesis and tissue fibrosis}

Upon injury or microvascular stress, Angpt1deficient mice displayed an excess of angio- genesis and tissue fibrosis (7). Previous studies have shown that systemic administration of a chimeric Angpt1 protein protects the kidney from diabetic microvascular damage (16). In the model proposed by Jeansson et al., loss of endogenous Angpt 1 aggravated the kidney glomerular damage in diabetes induced by streptozocin (7). The formation of the vascular basement membrane matrix was excessive in the Angpt1deficient diabetic glomeruli, presumably in response to the induction of TGF- $\beta$. Several lines of evidence suggest that the angiopoietins regulate endothelial cell-matrix interactions. Endothelial cell interactions with the subendothelial basement membrane are defective in the Angpt1-deleted embryos (8). Matrix-bound Angpt1 induces Tie2 localization to cell-matrix contacts in mobile (angiogenic) cells, mediating cell migration and matrix adhesion $(4,5)$. The disorganized glomerular basement membrane and detached endothelial cells observed in the glomeruli of the Angpt1deleted mice may have resulted from lack of such interaction between matrix-bound Angpt 1 and Tie 2 on the endothelial cells. Furthermore, several reports have suggest- ed that the angiopoietins bind to pericellular matrix and integrins and may even signal via the integrins $(2,17)$. This suggests that the Angpt 1 pathway provides a feedback sensor of the pericellular matrix - for example, in angiogenic sprouts. Further work should elucidate the exact mechanisms involved in these interactions.

Whether inflammatory cells contribute to the tissue fibrosis in the Angpt1-deleted mice remains to be studied. The fast release of Angpt 2 from Weibel-Palade bodies after endothelial activation by cytokines suggests a role for Angpt 2 in rapid control of vascular responses to inflammation (2). Indeed, in mice lacking Angpt2, leukocyte transmigration to inflamed tissues is defective (18). Although there were no compensatory changes in Angpt2 levels after Angpt1 knockout, the deletion would dramatically alter the Angpt1/Angpt2 ratio that is a critical switch controlling inflammatory processes, such as leukocyte transmigration in the vessel wall.

\section{The outstanding tumor questions}

The effects of Angpt1 deletion on tumor angiogenesis also remain to be studied. It 
has been suggested that Angpt1 is responsible for the tumor vessel normalization seen in patients treated with anti-VEGF antibodies (19). Again, the balance of Angpt1 and Angpt2 seems critical, as Angpt2 expression is known to be highly upregulated by endothelial cells of tumor co-opted blood vessels. This leads to vessel disruption and hypoxic induction of VEGF production and the initiation of angiogenesis (20). The angiopoietin system may be involved in the recruitment of proangiogenic myeloid cells and, in particular, Tie2-positive cells of the monocyte/macrophage lineage to tumors (21). In this regard, it is interesting that Angpt 1 has been shown to promote hematopoietic stem cell quiescence in the bone marrow (22), an activity that needs to be reassessed in the Angpt1-deleted mice. Angpt2-blocking molecules that have been successfully used to inhibit tumor angiogenesis seem to provide an additive inhibition over anti-VEGF therapy, and they are already in advanced clinical trials (23). It will be very interesting to know whether, according to the models, Angpt2 acts as an antagonist of Angpt 1 in the tumor vessels, a question that can now be addressed using the conditional Angpt1-knockout model. After all, a rational development of angiopoietin-based therapeutics depends on the general question of how Angpt2 and Angpt1, the yin and yang of angiogenesis, can induce both angiogenesis and vascular stabilization through their common receptor $(4,5)$.

\section{Acknowledgments}

The authors thank Hellmut Augustin, Donald McDonald, Lauri Eklund, and Gou
Young Koh for useful discussions and the Finnish Academy and Helsinki University Funds for continuous support.

Address correspondence to: Kari Alitalo, Molecular/Cancer Biology Program and Finnish Institute for Molecular Medicine, Biomedicum Helsinki, P.O.B. 63, 00014 University of Helsinki, Helsinki, Finland. Phone: 358.9.191.25511; Fax: 358.9.191.25510; E-mail: kari.alitalo@helsinki.fi.

1. Phng LK, Gerhardt H. Angiogenesis: a team effort coordinated by notch. Dev Cell. 2009;16(2):196-208.

2. Augustin HG, Koh GY, Thurston G, Alitalo K. Control of vascular morphogenesis and homeostasis through the angiopoietin-Tie system. Nat Rev Mol Cell Biol. 2009;10(3):165-177.

3. Maisonpierre PC, et al. Angiopoietin-2, a natural antagonist for Tie 2 that disrupts in vivo angiogenesis. Science. 1997;277(5322):55-60.

4. Fukuhara S, et al. Differential function of Tie2 at cell-cell contacts and cell-substratum contacts regulated by angiopoietin-1. Nat Cell Biol. 2008; 10(5):513-526.

5. Saharinen P, et al. Angiopoietins assemble distinct Tie2 signalling complexes in endothelial cellcell and cell-matrix contacts. Nat Cell Biol. 2008; 10(5):527-537

6. Wong AL, Haroon ZA, Werner S, Dewhirst MW, Greenberg CS, Peters KG. Tie2 expression and phosphorylation in angiogenic and quiescent adult tissues. Circ Res. 1997;81(4):567-574.

7. Jeansson M, et al. Angiopoietin- 1 is essential in mouse vasculature during development and in response to injury. J Clin Invest. 2011;121(6):2278-2289.

8. Suri C, et al. Requisite role of angiopoietin-1, a ligand for the TIE2 receptor, during embryonic angiogenesis. Cell. 1996;87(7):1171-1180.

9. Partanen J, et al. A novel endothelial cell surface receptor tyrosine kinase with extracellular epidermal growth factor homology domains. Mol Cell Biol. 1992;12(4):1698-1707.

10. Dumont DJ, et al. Dominant-negative and targeted null mutations in the endothelial receptor tyrosine kinase, tek, reveal a critical role in vasculogenesis of the embryo. Genes Dev. 1994;8(16):1897-1909.

11. Puri MC, Rossant J, Alitalo K, Bernstein A, Partanen J.
The receptor tyrosine kinase TIE is required for integrity and survival of vascular endothelial cells. EMBOJ. 1995;14(23):5884-5891.

12. Puri MC, Partanen J, Rossant J, Bernstein A. Interaction of the TEK and TIE receptor tyrosine kinases during cardiovascular development. Development. 1999;126(20):4569-4580.

13. Partanen J, Puri MC, Schwartz L, Fischer KD, Bernstein A, Rossant J. Cell autonomous functions of the receptor tyrosine kinase TIE in a late phase of angiogenic capillary growth and endothelial cell survival during murine development. Development. 1996; 122(10):3013-3021.

14. Fiedler $U$, et al. The Tie-2 ligand angiopoietin- 2 is stored in and rapidly released upon stimulation from endothelial cell Weibel-Palade bodies. Blood. 2004; 103(11):4150-4156.

15. Thurston G, et al. Angiopoietin-1 protects the adult vasculature against plasma leakage. Nat Med. 2000; 6(4):460-463.

16. Lee $S$, et al. Renoprotective effect of COMP-angiopoietin- 1 in $\mathrm{db} / \mathrm{db}$ mice with type 2 diabetes. Nephrol Dial Transplant. 2007;22(2):396-408.

17. Thomas M, et al. Angiopoietin-2 stimulation of endothelial cells induces alphavbeta 3 integrin internalization and degradation. J Biol Chem. 2010; 285(31):23842-23849.

18. Fiedler U, et al. Angiopoietin-2 sensitizes endothelial cells to TNF-alpha and has a crucial role in the induction of inflammation. Nat Med. 2006; 12(2):235-239.

19. Winkler F, et al. Kinetics of vascular normalization by VEGFR2 blockade governs brain tumor response to radiation: role of oxygenation, angiopoietin-1, and matrix metalloproteinases. Cancer Cell. 2004;6(6):553-563.

20. Holash J, et al. Vessel cooption, regression, and growth in tumors mediated by angiopoietins and VEGF. Science. 1999;284(5422):1994-1998.

21. Coffelt SB, Lewis CE, Naldini L, Brown JM, Ferrara N, De Palma M. Elusive identities and overlapping phenotypes of proangiogenic myeloid cells in tumors. Am J Pathol. 2010;176(4):1564-1576.

22. Arai $F$, et al. Tie2/angiopoietin-1 signaling regulates hematopoietic stem cell quiescence in the bone marrow niche. Cell. 2004;118(2):149-161.

23. Brown JL, et al. A human monoclonal anti-ANG2 antibody leads to broad antitumor activity in combination with VEGF inhibitors and chemotherapy agents in preclinical models. Mol Cancer Ther. 2010; 9(1):145-156 